\title{
A robust state of charge estimation for multiple models of lead acid battery using adaptive extended Kalman filter
}

\author{
Maamar Souaihia, Bachir Belmadani, Rachid Taleb \\ Electrical Engineering Department, Hassiba Benbouali University of Chlef, LGEER Laboratory \\ BP. 78C, Ouled Farès 02180, Chlef, Algeria
}

\begin{tabular}{l} 
Article Info \\
\hline Article history: \\
Received Jan 30, 2019 \\
Revised Aug 4, 2019 \\
Accepted Sep 26, 2019 \\
\hline
\end{tabular}

Keywords:

Adaptive Kalman filter Lead-acid batteries

Least square method

Open circuit voltage

State of charge es timation

\begin{abstract}
An accurate estimation technique of the state of charge (SOC) of batteries is an essential task of the battery management system. The adaptive Kalman filter (AEKF) has been used as an obsever to investigate the SOC estimation effectiveness. Therefore, The SOC is a reflexion of the chemistry of the cell which it is the key parameter for the battery management system. It is very complex to monitor the SOC and control the internal states of the cell. Three battery models are proposed and their state space models have been established, their parameters were identified by applying the least square method. However, the SOC estimation accuracy of the battery depends on the model and the efficiency of the algorithm. In this paper, AEKF technique is presented to estimate the SOC of Lead acid battery. The experimental data is used to identify the parameters of the three models and used to build different open circuit voltage-state of charge (OCV-SOC) functions relationship. The results shows that the SOC estimation based-model which has been built by hight order RC model can effectively limit the error, hence guaranty the accuracy and robustness.
\end{abstract}

This is an open access article under the CC BY-SA license.

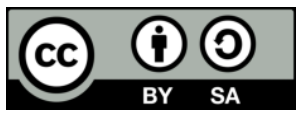

\section{Corresponding Author:}

Maamar Souaihia,

Department Electrical Engineering,

Hassiba Benbouali University of Chlef, LGEER Laboratory,

BP. 78C, Ouled Farès 02180, Chlef, Algeria.

Email: maa.souaihia@gmail.com

\section{INTRODUCTION}

Photovoltaic energy is the modern technology developed among the recent years due to the necessity of clean resource, safe energy with a cost reduced. The radiation of the sun flows on the solar panel, which makes the cell generate an electrical energy. The electrical energy converted from the cells is using to run stand-alone systems and many applications and also stored in the battery pack.

The conversion of the energy is an essential part in the solar systems by connecting the cells in some combination therefore to attain the necessary level of voltage and power. The battery man agement system is using to manage the electricity comes from solar panels, conduct operations by using different algorithms to achieve a high power whatever the radiation and the weather. Thus, it controls the charging process and manages the energy of the battery in discharge too. Therefore, it protects the whole system, controls and monitors the batteries, arranges the power between the system and the loads.

Energy storage systems are widely used in photovoltaic systems (PV), power grids, and electric vehicle. Lead-Acid batteries take over these utilizations due to its high energy and cycle of life [1]. In PV, a battery pack is a fundamental part, usually numerous battery cells connected in series or parallel, or even in mix technology hence, they employed to safeguard, ensure the operations at nights or emergency 
situations. Moreover, due the differences among the cells, finding the SOC for the whole pack is necessary. To ensure its proper running and extend the life of the battery, the key task is the estimation of the current status of the battery, which knows as the state of charge (SOC). Therefore, this paper investigates on the SOC estimation for the battery. The SOC, it is the reflexion ratio of the energy remaining on the battery, cannot be directly measured as it related to the chemical of the cell [2]. This is a challenge to use a capable algorithm for accurate estimating, by using measurable inputs, including the noise of sensors, temperature, and nonlinear behaviour.

State estimation is a recent subject, but it has been well explained in the last decade for reas on of electric vehicle and stand-alone emergency systems. There are numerous methods to determine the SOC [3, 4]. Current-based method (coulomb counting) [5, 6] use the equation of drawn current from the battery capacity to estimate SOC. It is a less accurate method, thus the initial SOC must be known and that is not always possible. Moreover, the accumulated current error measurement may cause inaccurate estimation. Voltage-based methods [7-9] use the relationship between open circuit voltage (OCV) and SOC. The battery has to be rested enough time to reach the equilibrium state. Therefore, the relationship suffers from changes with different effects. Model-based estimation [10-12] uses mathematical models to link measured signals, it is known to done accurate and robust estimates. It is based on the equivalent circuit models.

SOC estimation using model-based, an accurate battery model is most important; a model suitable of capturing and tracking the battery dynamics and also easy to implement. Two general types of classification; Electrochemical models [13] use the chemical equations to describe the electrochemical process, they represent the battery behaviour accurately but they are complex to implement and quivalent circuit models (ECM) $[14,15]$ use electrical components to describe electrochemical processes like resistors and capacitors. ECMs are commonly used due to their simplicity in implementation and accuracy of estimation.

In [16-18] proposed ECM involved of Shepherd, Unnewher and Nernst models. Other models like hysteresis and self-correcting are presented, the accuracy of these models can be improved by adding RC branches. In [19] a comprehensive study is presented with determination of the parameter. Thus, different models have been used. Among them, the second order RC model has been given well results. Since, the estimation algorithm use measurements to construct the internal states, by the usage of observers. The extended Kalman filter is the common choice to deal with nonlinear systems [20]. In three papers [17, 18, 21] Gregory Plett, presented the EKF algorithm and investigate the accuracy of SOC estimation with battery model and explained the limitation of it. In both paper; states estimation with time varying parameters gave good results. Also SOC estimation using Kalman filter is investigated in [22-24] showed performance however the scenarios, also the unscented Kalman filter [25] (UKF) performs relatively better. In [19] the comparison between 18 different Kalman filter showed the dual EKF was found to perform the best.

This paper investigates of the SOC estimating for a lead acid battery with adaptive Kalman filter. The battery model is critical for SOC estimation, therefore three model namely first order equivalent circuit, second order and third order model are considered, combined with four OCV-SOC functions in term to modelling accuracy. The paper is organized as follows: Section 2 introduces the battery models. In section 3, the method has been used to identify models parameters, the AEKF algorithm briefly introduced while this is followed by experiment results and analysis. Finally, conclusions of the paper are given in section 5.

\section{BATTERY MODELLING}

In order to apply a model based to achieve an accurate SOC estimation, firstly, a battery model should be designed and constructed. Thus, equivalent circuit model (ECM) equations required to build and estimate the parameters and the states. Therefore, numerous types of battery models have been used to describe the dynamics of the battery were presented and evaluated [1]. To reach excellent capture between the output voltage and the characteristics of the battery considering the complexity of computation and speed response, three models has been selected as illustrated in Figure 1.

The first order model consists of an open circuit voltage (OCV), a series resistance $\mathrm{R}_{\mathrm{s}}$ represents the internal resistance of the cell which denotes the resistivity of the electrolyte, the separator and electrodes. In addition to a parallel branch of resistance and capacitance polarization connected in series. While, the second order model contains two RC branches and the third order model contains three RC branches. $\mathrm{I}_{\mathrm{L}}$ expresses the load current (it takes negative for charge, positive for discharge), $V_{t}$ indicates the terminal voltage. The OCV is used to denote the internal voltage source of the battery. The electrical behaviour of three proposed model a,b and c can be written in (1), (2) and (3) respectively: 
Model (a)

$$
\left\{\begin{array}{c}
\frac{d V_{1}}{d t}=\frac{-\mathrm{V}_{1}}{R_{1} C_{1}}+\frac{I_{L}}{\mathrm{C}_{1}} \\
V_{t}=\operatorname{OCV}(z)-I_{L} R_{0}-V_{1}
\end{array}\right.
$$

$$
\left\{\begin{array}{c}
\frac{d V_{1}}{d t}=\frac{-\mathrm{V}_{1}}{R_{1} C_{1}}+\frac{I_{L}}{\mathrm{C}_{1}} \\
\frac{d V_{2}}{d t}=\frac{-\mathrm{V}_{2}}{R_{2} C_{2}}+\frac{I_{L}}{\mathrm{C}_{2}} \\
V_{t}=\operatorname{OCV}(z)-I_{L} R_{0}-V_{1}-V_{2}
\end{array}\right.
$$

Model (c)

$$
\begin{aligned}
\frac{d V_{1}}{d t} & =\frac{-\mathrm{V}_{1}}{R_{1} C_{1}}+\frac{I_{L}}{\mathrm{C}_{1}} \\
\frac{d V_{2}}{d t} & =\frac{-\mathrm{V}_{2}}{R_{2} C_{2}}+\frac{I_{L}}{\mathrm{C}_{2}} \\
\frac{d V_{3}}{d t}= & \frac{-\mathrm{V}_{3}}{R_{3} C_{3}}+\frac{I_{L}}{\mathrm{C}_{3}} \\
V_{t}=O C V(z) & -I_{L} R_{0}-V_{1}-V_{2}-V_{3}
\end{aligned}
$$

Where $\mathrm{z}$ denotes the state of charge attached to each polarization voltage.

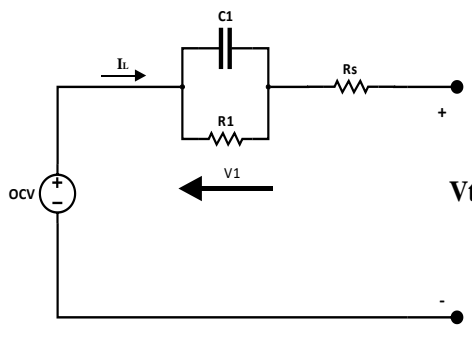

(a)

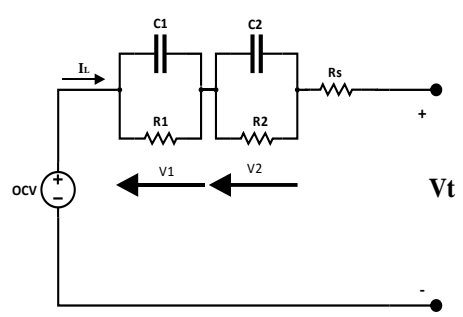

(b)

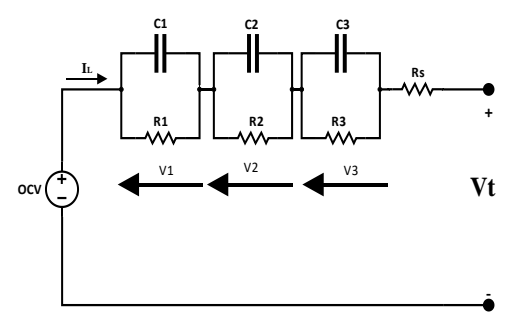

(c)

Figure 1. (a) The first order Thevenin model, (b) The second order RC model, (c) The third order RC model

\section{PARAMETERS IDENTIFICATION}

Before the implementation of the proposed approach to estimating the state, first, the identification of the parameters which the model contains is recommended. In order to determine the parameters of the model a pulse discharge method is applied. A test bench settles in to figure out these factors, a lead-acid battery is carried out in this experiment with a nominal voltage and capacity of $12 \mathrm{~V}$ and $22 \mathrm{Ah}$ respectively. The OCV curve can be obtained based on the voltage measurement after the battery reaches its balance voltage. In this paper, the battery is fully charged and has been left a period to get its equilibrium, we measure the OCV at the beginning of the test. Then the cell discharged of $10 \%$ capacity with a current of $1 \mathrm{C}$, and let it in a relaxation period. We measure the OCV value with the 0.9 SOC, the steps can be repeated until the battery is fully discharged.

It is clearly appearing that the main objective of this test is to excite the dynamics of the cell for a pulse discharge. The terminal voltage and current curves are illustrated in Figure 2. The terminal voltage 
interval from around $12.83 \mathrm{~V}$ to $10.5 \mathrm{~V}$ when the pulse discharged applied, but it can be observed that returns back to 11 after relaxation.

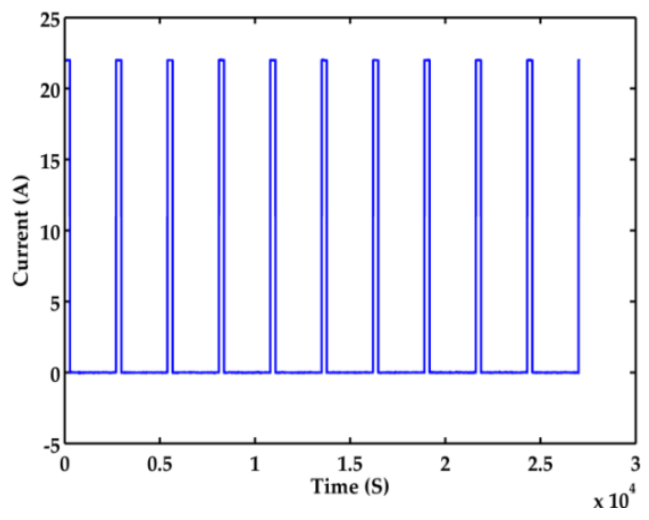

(a)

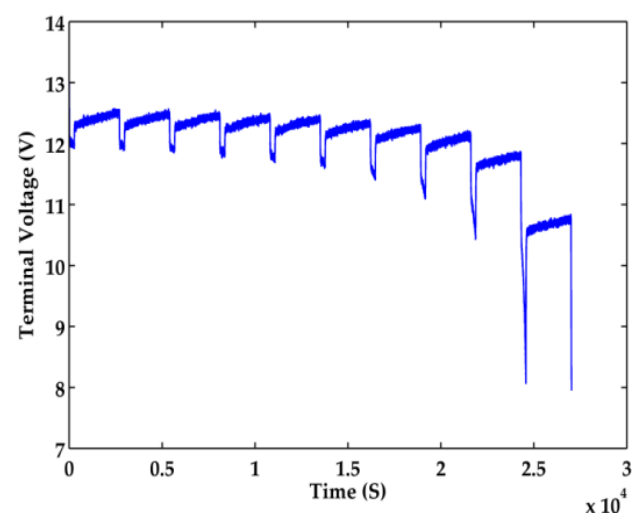

(b)

Figure 2. Pulse discharge, (a) Current $1 \mathrm{C}$ rate, (b) Terminal voltage measured

\subsection{Electrical model parameters}

Based on the curve fitting tool in MATLAB, an equation of six-order polynomial built by the measured data is employed to simulate the open circuit voltage, as shown in Figure 3. Before applying the approaches technique for the SOC prediction, the state transition and measurement update equations that link the SOC to the terminal voltage based on the equivalent circuit model necessary to be built firstly. The transient response voltage for a period a pulse discharge is presented in Figure 4(a). The components of the equivalent circuit model can be identified from the transient voltage. The voltage across the branch RC for the loaded in and the relaxation period is written in (4) and (5) as mentioned the Figure 4(b).

$$
\mathrm{R}_{0}=\left(\left|\frac{\mathrm{V}_{1}-\mathrm{V}_{2}}{\mathrm{I}_{\mathrm{L}}}\right|+\left|\frac{\mathrm{V}_{3}-\mathrm{V}_{4}}{\mathrm{I}_{\mathrm{L}}}\right|\right) / 2
$$

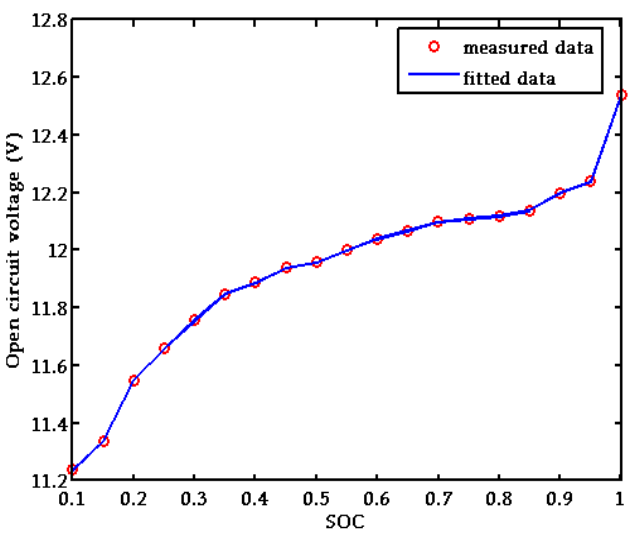

Figure 3. The relationship between open circuit voltage and SOC

The transient parameters can be identified from the present equation for the first model by using LS method [26]:

$$
\begin{cases}\mathrm{V}_{\mathrm{d}}=\mathrm{I}_{\mathrm{L}} * \mathrm{R}_{\mathrm{p}} *\left[1-\exp \left(\frac{-\mathrm{t}_{\mathrm{d}}}{\tau}\right)\right] & , \mathrm{I}_{\mathrm{L}} \neq 0 \\ \mathrm{~V}_{\mathrm{d}}=\mathrm{V}_{\mathrm{d}} *\left[\exp \left(\frac{-\mathrm{t}_{\mathrm{r}}}{\tau}\right)\right] & , \mathrm{I}_{\mathrm{L}}=0\end{cases}
$$


Where, $I_{L}$ denotes the discharge current, $t_{d}$ is the time of the discharge period and $t_{r}$ is the time of the rest period for the model(a) as illustrated in Figure 1(a). And similarly, the transient parameters for the (b) and (c) models can be identified by adding the second branch transient and third transient as illustrate in the Figures 1(b) and Figure 1(c).

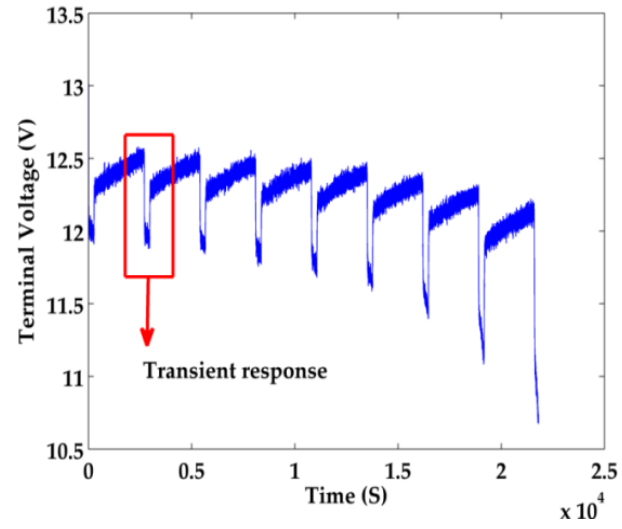

(a)

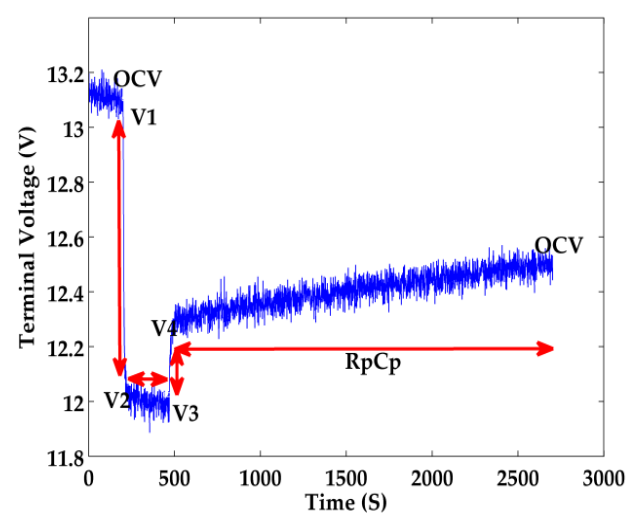

(b)

Figure 4. The parameters identification method for Theven in method, (a) Voltage response, (b) Zoomed transient response

\subsection{The state of charge}

The SOC is defined in the literature as a ratio refers to the remaining energy in the battery. It indicated in the range of 0 and 1 or expressed in percentage. The SOC function given by:

$$
S O C_{t}=S O C_{0}-\frac{1}{C_{n}} \int_{0}^{t} n_{i} I_{L, \tau} d \tau
$$

Where $S O C_{t}$ is the current time. $S O C_{0}$ is the initial value of the $S O C, I_{L}$ is the current flow in the load assumed negative for charge and positive of discharge. $C_{n}$ is the present available capacity, which may change by the age effect. $n_{i}$ refers to the coulomb efficiency. Afterwards, the equation can be transformed to the discrete time form as:

$$
S O C_{k}=S O C_{k-1}-n_{i} \frac{I_{L, k} \Delta t}{C_{n}}
$$

Where $\Delta t$ is the sampling time.

\subsection{AEKF algorithm}

Several models linked to the battery, such as electrochemical models and ECMs have been widely used due to the battery system belongs to the nonlinear systems. The version of Kalman filter which is designed to deal with such kind of systems is the extended Kalman filter [27]. It is a recursive filter based on mathematical equations for estimating the states of a process, designed to minimize the mean squared error. It has been used in many fields as tracking and estimating. The main goal to apply AEKF because it can iteratively regulate the gain automatically based on the update of the weight noise coefficient and adaptively adjusts the output model with the measured voltage. The state space equations of the three models can be formulated and presented as [28-30]:

$$
\left\{\begin{array}{c}
x_{k}=A_{k} x_{k-1}+B_{k} u_{k-1}+w_{k} \\
y_{k}=C_{k} x_{k}+D_{k} u_{k}+v_{k}
\end{array}\right.
$$

where $v_{k}, v_{k}$ are the process and measurement noise with zero mean Gaussian respectively of the nonlinear functions [27, 29] $f($.$) and g($.$) . Therefore, based on the equivalent circuit of the three selected batteries$ models, $u_{k}$ is selected as the input current and the output of the system $y_{k}$ is the terminal voltage. $x_{k}$ is the systemstates; here are the matrices of the systemas follow in (9) for the three models respectively. 


$$
\begin{aligned}
& \text { Model (a) } \\
& \text { Model (b) }
\end{aligned}
$$

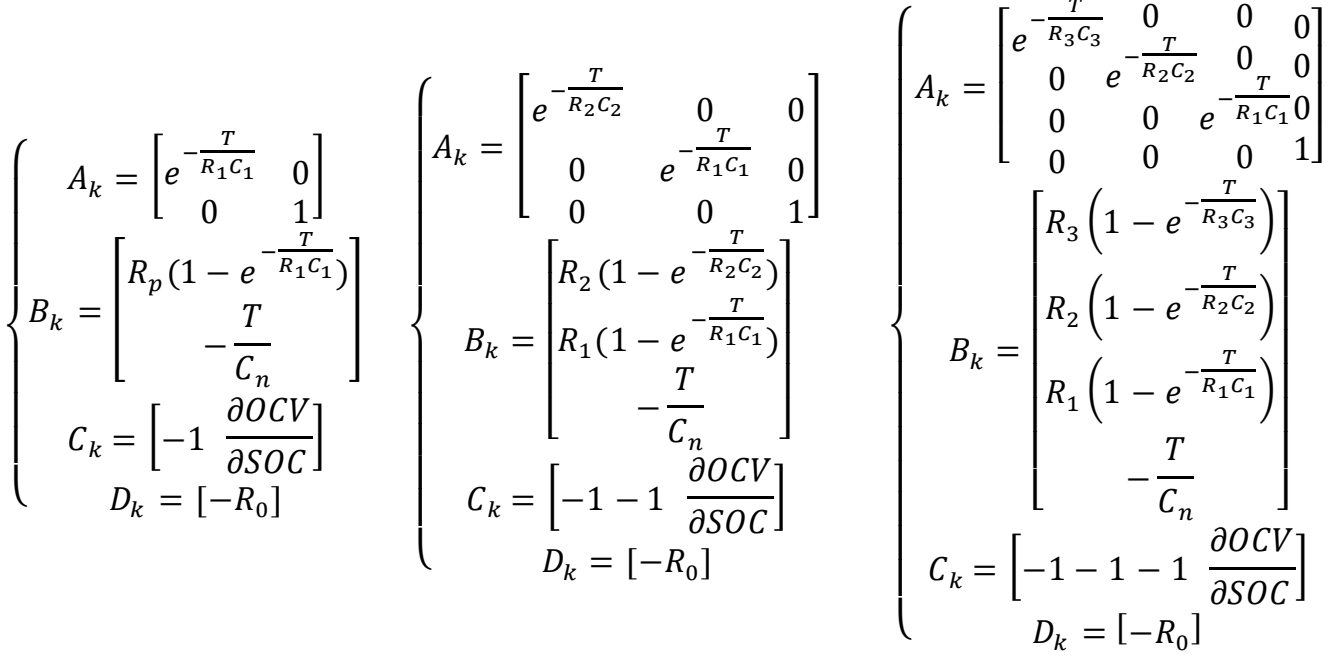

Where $A_{k}$ and $B_{k}$ are discrete system matrix and discrete input matrix for system, respectively $T$ is the sampling time with 1 second, and $k$ is the step. Their covariance values are denoting as $Q_{k}$ and $R_{k}$ respectively to the process noise and measurement noise $w_{k}$ and $v_{k} . C_{n}$ is the capacity of the battery supposed to be constant. Minimizing the mean square error is the base of the AEKF algorithm. It is applying the recursive estimation, involved of the initialization, time update and measurement update. The process is illustrated in Figure 5.

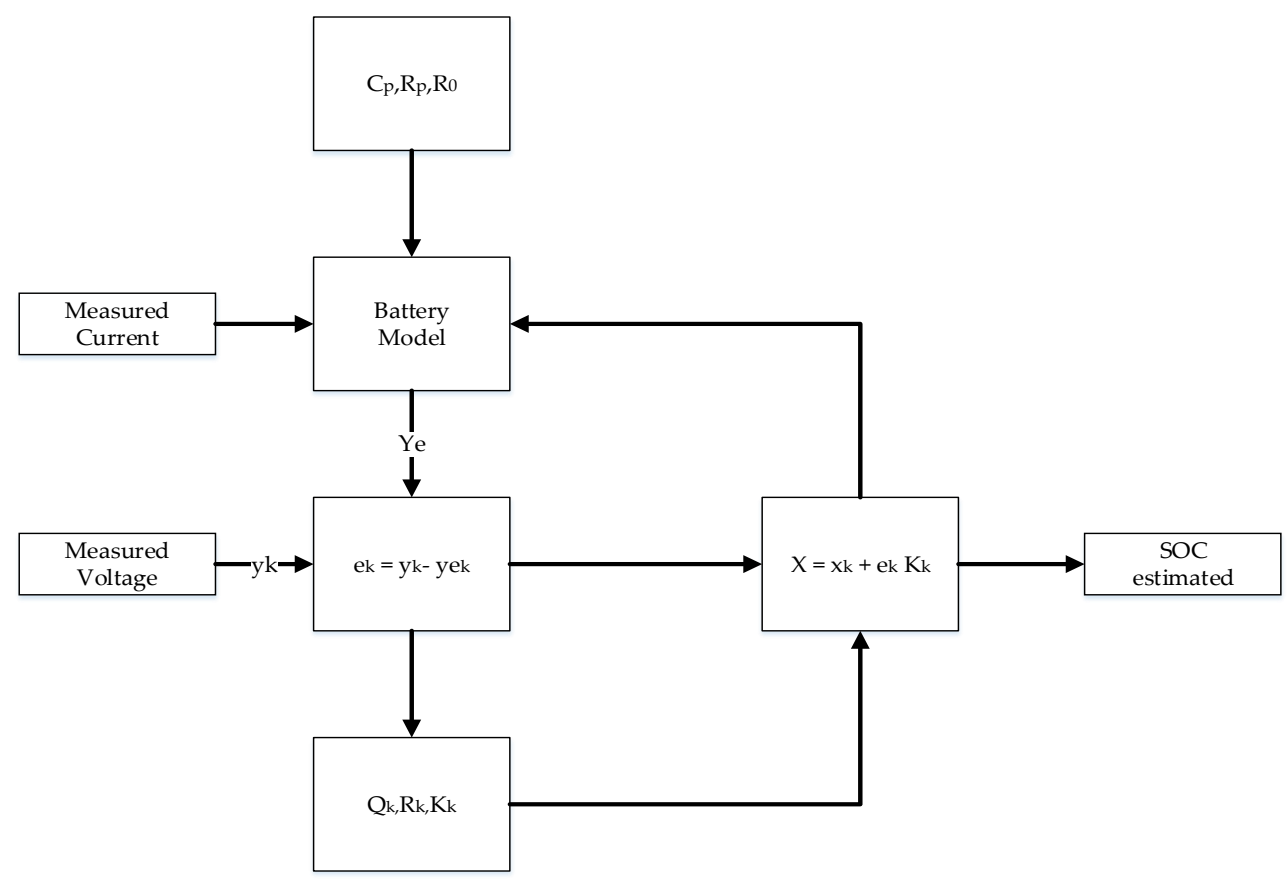

Figure 5. Structure of the process of AEKF

And the steps of the AEKF are summarized as follows:

a. Initialization of parameters:

$$
\left\{\begin{array}{c}
x_{0}=E\left(x_{0}\right) \\
P_{0}=\operatorname{var}\left(x_{0}\right)
\end{array}\right.
$$

Where, $E$ and var are the mean value of state variable and the initial covariance of the system, respectively, The calculation of AEKF consists of six steps. 
b. Innovation:

$$
e_{k}=y_{k}-g\left(\hat{x}_{k}^{-}, u_{k}\right)
$$

c. Adaptive law:

The covariance has been iteratively updating. $Q_{k}$ and $R_{k}$ have been estimated respectively, which cannot estimate accurately in EKF calculation process.

$$
\begin{aligned}
& H_{k}=\frac{1}{M} \sum_{k-M+1}^{k} e_{i} e_{i}^{T} \\
& R_{k}=H_{k}-C_{k} P_{k}^{-} C_{k}^{T}
\end{aligned}
$$

$H_{k}$ is the computation which states covariance of $e_{k}$ with a moving estimation windows of size M.

d. State estimation covariance and Kalman gain

$$
\begin{aligned}
& P_{K}^{-}=\left(I+A_{k} \Delta t\right) P_{k-1}\left(I+A_{k} \Delta t\right)^{T}+Q_{k} \\
& K_{k}=P_{k}^{-} C_{k}^{T} *\left(C_{k} P_{k}^{-} C_{k}^{T}+R_{k}\right)^{T}
\end{aligned}
$$

e. State estimate update

$$
\hat{x}_{k}^{+}=\hat{x}_{k}^{-}+K_{k} e_{k}
$$

f. Update state covariance

$$
\begin{aligned}
& Q_{k}=K_{k}^{-} H_{k} K_{k} \\
& P_{k}^{+}=\left(I-K_{k} C_{k}\right) P_{k}^{-}\left(I-K_{k} C_{k}\right)^{T}+K_{k} R_{k} K_{k}^{T}
\end{aligned}
$$

In the final step, error covariance is estimated and updated. The computing is then repeating again from (b) to (f) until reach all the collected data sampling [30].

\section{RESULTS AND SIMULATION}

In this practical test, the actual battery's capacity is ranging in the level of $10 \%-95 \%$, to protect it from damage. The data were used for simulation area, were chosen in order to get the constant discharge curve illustrated in Figure 4. Thus, From Figure 4, it appears that The OCV-SOC relationship drawn is a nonlinear function. In order to investigate the nonlinear behaviour, 4 different functions [17,31] for the OCV proposed to fit the OCV-SOC curve. The functions descriptive are shown in Table 1. Moreover, their parameters were obtained from the curve fitting toolbox in Matlab and evaluated their goodness of fitting.

Table 1. Candidates' functions fitting

\begin{tabular}{cc}
\hline Function & Function descriptive \\
\hline Func1 & $O C V=a_{1} S O C^{6}+a_{2} S O C^{5}+a_{3} S O C^{4}+a_{4} S O C^{3}+a_{5} S O C^{2}+a_{6} S O C+a_{7}$ \\
Func2 & $O C V=a_{1} S O C^{5}+a_{2} S O C^{4}+a_{3} S O C^{3}+a_{4} S O C^{2}+a_{5} S O C^{1}+a_{6}$ \\
Func3 & $O C V=a_{1}+\frac{a_{2}}{S O C}+a_{3} S O C+a_{4} \ln (S O C)+a_{5} \ln (1-S O C)$ \\
Func4 & $O C V=a_{1}+\frac{a_{2}}{S O C}+a_{3} S O C^{2}+a_{4} \mathrm{e}^{-a_{5}(1+S O C)}$ \\
\hline
\end{tabular}

The obtained parameters after fitting the curve with each candidate function are shown in Table 2. Followed by the root mean of the sum error (RMSE) for each candidate function, the results are shown in Table 3.

Table 2. Parameters of the selected functions

\begin{tabular}{cccccccc}
\hline OCV function & $a_{1}$ & $a_{2}$ & $a_{3}$ & $a_{4}$ & $a_{5}$ & $a_{6}$ & $a_{7}$ \\
\hline Func1 & 142 & -439.3 & 528.7 & -308.7 & 86.69 & -8.2 & 11.45 \\
Func2 & 24.88 & -62.01 & 60.09 & -30.04 & 9.076 & 10.54 & - \\
Func3 & 13.7 & 0.1009 & -2.098 & 1.428 & -0.1778 & - & - \\
Func4 & 6.406 & 0.02342 & -12.69 & 0.8754 & -1.532 & - & - \\
\hline
\end{tabular}


Table 3. RMSE after fitting

\begin{tabular}{ccccc}
\hline OCVfunction & Func1 & Func2 & Func3 & Func4 \\
\hline RMSE & 0.0225 & 0.0377 & 0.0203 & 0.044 \\
\hline
\end{tabular}

The test was settled first to test the performance of the model-based proposed with discharging of the battery under a constant current. The results obtained are shown in Figure 6(a) and Figure 6(b), in comparison with the terminal voltage obtained from the model. Choosing the right OCV function is the key to better estimation the SOC of the battery. Better estimation results were getting when using logarithm, exponential and polynomial functions. The better performance of the higher polynomial function, it is much better than that other OCV functions in the model-based on the first order Thevenin model as shown in Figure 7(a). In Figure 8(a), it can be seen that the SOC error less than the previous shown in Figure7(b). The highest polynomial function fits better than the other functions, and the result become better on tracking by using the second order Thevenin model.

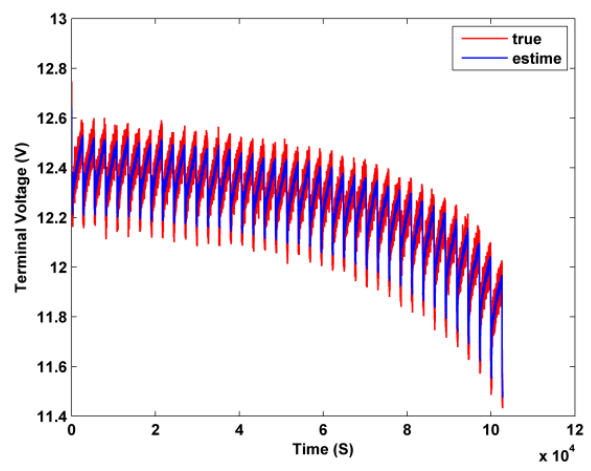

(a)

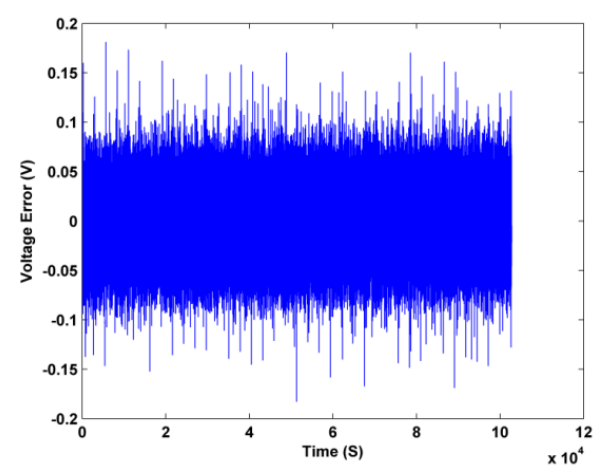

(b)

Figure 6. The terminal voltage modelled: (a) the modelling voltage; (b) the modelling error

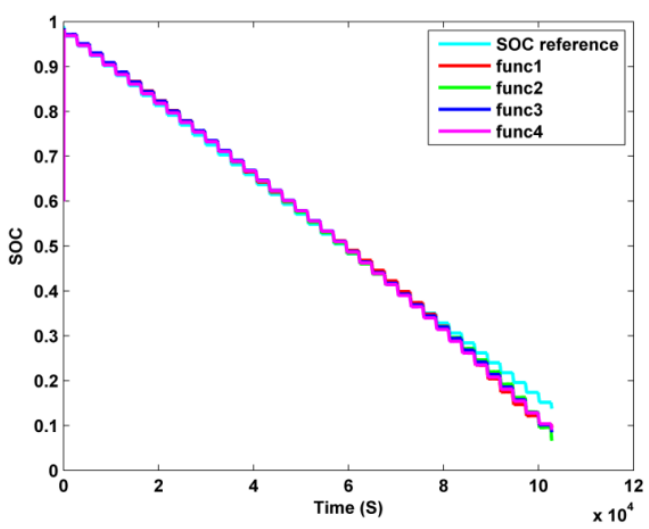

(a)

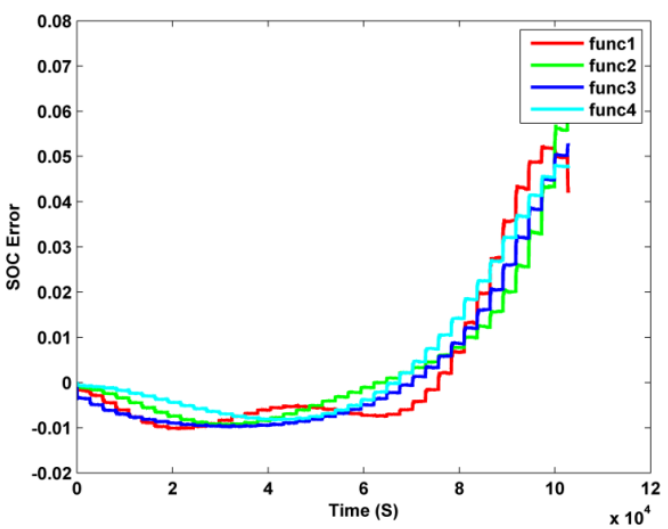

(b)

Figure 7. The SOC estimation with initial value of $60 \%$, (a) SOC estimation for first order Thevenin model,

(b) The SOC estimation error

In Figure 8(b), it shows the SOC tracking become well than the previous results seen in Figure 8(a) and Figure 7(b). Also the initial SOC given is corrected; thus, it took some period of time to get the correction in addition, the RMSE for each function with the third ECM model. Although the Kalman filter suffered from the initialization error. However, the EKF perform better with the third order model and the high polynomial function of the OCV even when the model was affected by the noise. The RMSE for the SOC estimated are tabulated in Table 4. 


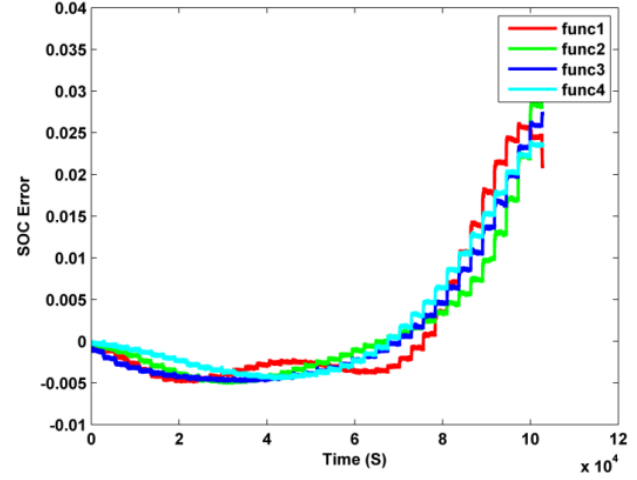

(a)

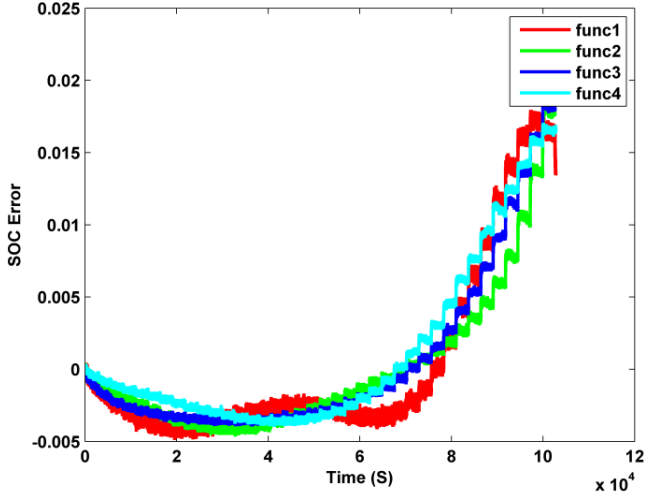

(b)

Figure 8. The SOC estimation error, (a) For second-order model, (b) For third-order model

Table 4. The RMSE of SOC estimated for third model-based

\begin{tabular}{ccccc}
\hline Function & Func1 & Func2 & Func3 & Func4 \\
\hline RMSE & 0.0144 & 0.0168 & 0.0193 & 0.0201 \\
\hline
\end{tabular}

The state of charge of the battery can be estimated with sliding mode observer (SMO) algorithm. This solution is simple and optimal for estimating the SOC. So, considering the battery system with the sliding variable $V_{t}$ and the error defined as the sliding surface $e(t)=V_{t}-\widehat{V}_{t}$, where $V_{t}$ and $\widehat{V}_{t}$ are the terminal voltage and the terminal voltage estimate. The SMO is modeled as:

$$
\left\{\begin{array}{c}
\hat{\dot{x}}=f(\hat{x}, u)+k * \operatorname{sgn}(y-\hat{y}) \\
\hat{y}=C(\hat{x})
\end{array}\right.
$$

where sgn is signum function. $k$ is the switching gain. $y$ and $\hat{y}$ denote the true terminal voltage and estimated terminal voltage $V_{t}, \widehat{V}_{t}$, respectively. $u$ is the input control, which represent the current $I_{L}$. $\hat{x}$ is the estimated states. $C$ is the matrix of control.

Therefore, the AEKF has shown better tracking capability to the real SOC against conventional SMO, also has a smooth error during the estimation, which means suitable and robustness technique as shown in Figure 9. Moreover, the RMSE of AEKF equal 0.018 and SMO 0.021, but the SMO is the speed one by computationale time under $0.2 \mathrm{~S}$.
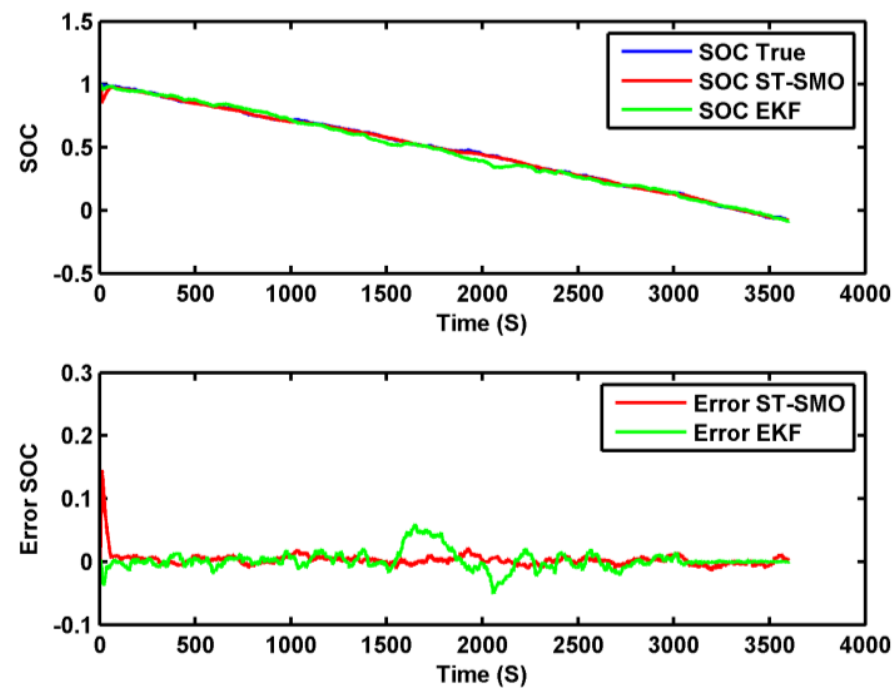

Figure 9. Comparison between AEKF and SMO 


\section{CONCLUSION}

This paper lies in development and testing of an algorithm of SOC estimation for a wide range of operational scenarios. There exists a difficulty in limitation of the performance of the Kalman filtering algorithm because of the nonlinearity in such systems. Thus, It is hard to acquiring suitable battery system data for several profiles. In this paper, an experimental data has been simulated for the model of a Lead-Acid battery to test the algorithm with different drive cycles and compared to simulated ones. The state space model of the battery and its parameters firstly determined and followed by applying the AEKF. The SOC estimation with four different candidate functions was settled. The combination of the EKF with the highest polynomial function and the third order model performed better in limitation of the error. The EKF proved to be more effective and accurate.

\section{REFERENCES}

[1] Plett GL., "Battery management systems," Volume II: Equivalent-circuit methods. vol. 2. Artech House; 2015.

[2] Lu L, Han X, Li J, Hua J, Ouy ang M., "A review on the key issues for lithium-ion battery management in electric vehicles," J Power Sources. vol. 226, pp. 272-288, 2013.

[3] Di-Domenico D, Creff Y, Prada E., "A review of approaches for the design of Li-ion BMS estimation functions," IFP Energies Nouv-RHEVE 2011. 2011; (December):1-8.

[4] Plett GL., "Battery management systems," Volume I: Battery Modeling [Internet]. Artech House; 2015. (Artech House power engineering series).

[5] W. Huihui and Z. Hongpeng, "SOC estimation and simulation of electric vehicle lead-acid storage battery with Kalman filtering method," 2013 IEEE 11th International Conference on Electronic Measurement \& Instruments, Harbin, 2013, pp. 599-603.

[6] Ng K. S., Moo C. S., Chen Y. P., Hsieh Y. C., "Enhanced coulomb counting method for estimating state-of-charge and state-of-health of lithium-ion batteries," Appl Energy, vol. 86, no. 9, pp. 1506-1511, 2009.

[7] Jackey R, Saginaw M, Sanghvi P, Gazzarri J, Huria T, Ceraolo M., "Battery model parameter estimation using a layered technique: an example using a lithium iron phosphate Cell," 2013; Available from: http://papers.sae.org/2013-01-1547/

[8] Feng F, Lu R, Wei G, Zhu C., "Online estimation of model parameters and state of charge of LiFePO4 batteries using a novel open-circuit voltage at various ambient temperatures," Energies, vol. 8, no. 4, pp. 2950-2976, 2015.

[9] K. Ng, C. Moo, Yi-Ping Chen and Y. Hsieh, "State-of-charge estimation for lead-acid batteries based on dynamic open-circuit voltage," 2008 IEEE 2nd International Power and Energy Conference, Johor Bahru, 2008, pp. 972-976.

[10] Min Chen and G. A. Rincon-Mora, "Accurate electrical battery model capable of predicting runtime and I-V performance," in IEEE Transactions on Energy Conversion, vol. 21, no. 2, pp. 504-511, June 2006.

[11] Y. Sun, H. Jou and J. Wu, "Intelligent aging estimation method for lead-acid battery," 2008 Eighth International Conference on Intelligent Systems Design and Applications, Kaohsiung, 2008, pp. 251-256.

[12] Zhao T, Jiang J, Zhang C, Bai K, Li N., "Robust online state of charge estimation of lithium-ion battery pack based on error sensitivity analysis," Math Probl Eng. 2015.

[13] T. He, D. Li, Z. Wu, Y. Xue and Y. Yang, "A modified luenberger observer for SOC estimation of lithium-ion battery," 201736th Chinese Control Conference (CCC), Dalian, 2017, pp. 924-928.

[14] Xiao R, Shen J, Li X, Yan W, Pan E, Chen Z. "Comparisons of modeling and state of charge estimation for lithiumion battery based on fractional order and integral order methods," Energies, vol. 9, no. 3, pp. 1-15, 2016.

[15] B. Xiao, Y. Shi and L. He, "A universal state-of-charge algorithm for batteries," Design Automation Conference, Anaheim, CA, 2010, pp.687-692.

[16] Plett GS., "Kalman-filter SOC estimation for LiPB HEV cells," CD-ROM Proc 19th Electr Veh Symp., pp. 1-12, 2002.

[17] Plett GL., "Extended Kalman filtering for battery management systems of LiPB-based HEV battery packs: Part 3. State and parameter estimation," J Power Sources, vol. 134, no. 2, pp. 277-292, 2004.

[18] Plett GL., "Sigma-point Kalman filtering for battery management systems of LiPB-based HEV battery packs: Part 2: simultaneous state and parameter estimation," J Power Sources, vol. 161, no. 2, pp. 1369-1384, 2006.

[19] Campestrini C, Heil T, Kosch S, Jossen A., "A comparative study and review of different Kalman filters by apply ing an enhanced validation method," J Energy Storage, vol. 8, pp. 142-159, 2016.

[20] Fei Zhang, Guangjun Liu and Lijin Fang, "A battery state of charge estimation method with extended Kalman filter," 2008 IEEE/ASME International Conference on Advanced Intelligent Mechatronics, Xian, 2008, pp. 1008-1013.

[21] Plett GL., "Dual and joint EKF for simultaneous SOC and SOH estimation," in: Proceedings of the 21st Electric Vehicle Symposium (EVS21), Monaco. 2005. pp. 1-12.

[22] R. Yamin and A. Rachid, "Modeling and simulation of a lead-acid battery packs in M ATLAB/simulink: parameters identification using extended Kalman filter algorithm," 2014 UKSim-AMSS 16th International Conference on Computer Modelling and Simulation, Cambridge, 2014, pp.363-368.

[23] Sepasi S, Roose LR, Matsuura MM. "Extended Kalman filter with a fuzzy method for accurate battery pack state of charge estimation". Energies, vol. 8, no. 6, .pp. 5217-5233, 2015. 
[24] Codecà F, Savaresi SM, Manzoni V, Di-Domenico D, Creff Y, Prada E, et al. "Battery state-of-charge estimating using adaptive extended Kalman filter with fuzzy modelling of the nominal battery capacity key words". Energies, vol. 1, no. 2, pp. 1-8, 2013.

[25] C. Piao, Z. Sun, Z. Liang and C. Cho, "SOC estimation of lead-acid batteries based on UKF," 2010 International Conference on Electrical and Control Engineering, Wuhan, pp. 1968-1972, 2010.

[26] Thompson EH. The theory of the method of least squares. Photogramm Rec, vol. 4, no. 19, pp. 53-65, 1962.

[27] He H, Xiong R, Guo H. "Online estimation of model parameters and state-of-charge of LiFePO4batteries in electric vehicles". Appl Energy, vol. 89, no. 1, pp.413-420, 2012.

[28] F. Codecà, S. M. Savaresi and V. Manzoni, "The mix estimation algorithm for battery state-of-charge estimatoranalysis of the sensitivity to measurement errors," Proceedings of the 48h IEEE Conference on Decision and Control (CDC) held jointly with 2009 28th Chinese Control Conference, Shanghai, 2009, pp. 8083-8088.

[29] S. C. L. da Costa, A. S. Araujo and A. d. S. Carvalho, "Battery state of charge estimation using extended Kalman filter," 2016 International Symposium on Power Electronics, Electrical Drives, Automation and Motion (SPEEDAM), Anacapri, pp. 1085-1092, 2016

[30] Xiong R, Gong X, Mi CC, Sun F. "A robust state-of-charge estimator for multiple types of lithium-ion batteries using adaptive extended Kalman filter". J Power Sources, vol. 243, pp. 805-816, 2016.

[31] Tian Y, Xia B, Wang M, Sun W, Xu Z. "Comparison study on two model-based adaptive algorithms for SOC estimation of lithium-ion batteries in electric vehicles," Energies, vol. 7, no. 12, pp. 8446-8464, 2014.

\section{BIOGRAPHIES OF AUTHORS}

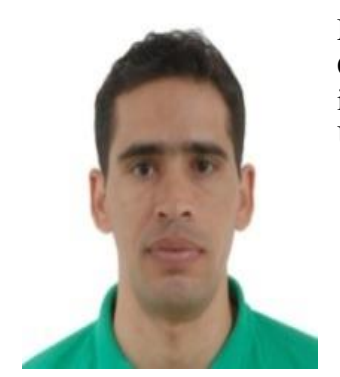

Maamar Souaihia received his engineering bachelor degree in electronics from University of Chlef in 2010 and his master degree in the industrial computing from University of Mostaganem in 2014. He is preparing his PhD thesis on the subject analy sis and diagnosis batteries since 2014 University of Chlef. E-mail: m.souaihia@univ-chlef.dz

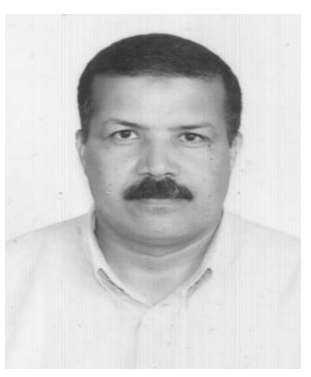

Bachir Belmadani received the B.S. degree in Electrical Engineering from University of Oran, in 1985, and the PhD degree from the Paul Sabatier University of Toulouse, France, in 1989. He is currently Professor of Power Electronics in Chlef University, Algeria. He is the Director of the Laboratory of Electrical Engineering and Renewable Energy. E-mail: belmadanibachir@gmail.com

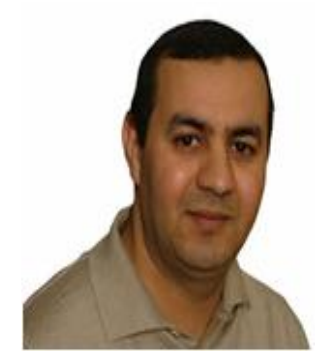

Rachid Taleb received the M.S. degree in electrical engineering from the Hassiba Benbouali University, Chlef, Algeria, in 2004 and the Ph.D. degree in electrical engineering from the Djillali Liabes University, Sidi Bel-Abbes, Algeria, in 2011. Currently he is a professor with the Department of Electrical Engineering, Hassiba Benbouali University. He is a team leader in the LGEER Laboratory (Laboratoire Génie Electrique et Energies Renouvelables). His research interest includes intelligent control, heuristic optimization, control theory of converters and converters for renewable energy sources. E-mail: rac.taleb@gmail.com 